\title{
Fatal Familial Insomnia
}

National Cancer Institute

\section{Source}

National Cancer Institute. Fatal Familial Insomnia. NCI Thesaurus. Code C84711.

A very rare autosomal dominant inherited sleep disorder caused by a mutation in the gene responsible for the prion protein. It affects individuals usually in their fourth decade. Its initial manifestation is difficulty in falling asleep. It is followed by complete inability to sleep. Patients develop deterioration of their mental and motor functions and die soon after the first symptoms appear, because of the total absence of sleep. 